\title{
Hookworm Infection Caused Acute Intestinal Bleeding Diagnosed by Capsule: A Case Report and Literature Review
}

\author{
Xia Tan', Meichu Cheng', Jie Zhang'2, Guochun Chen'1, Di Liu', Yexin Liu', Hong Liu',* \\ ${ }^{1}$ Department of Nephrology, ${ }^{2}$ Department of Gastroenterology, The Second Xiangya Hospital of Central South University, Renal Research Institute of \\ Central South University, Key Laboratory of Kidney Disease and Blood Purification in Hunan, Changsha 410011, Hunan, P. R. China
}

\begin{abstract}
Hookworm infections are rare causes of acute gastrointestinal bleeding. We report a middle aged man with primary nephrotic syndrome and pulmonary embolism. During the treatment with steroids and anticoagulants, the patient presented acute massive hemorrhage of the gastrointestinal tract. The results of gastroscopy showed red worms in the duodenum. Colonoscopy and CT angiogram of abdomen were unremarkable. Capsule endoscopy revealed fresh blood and multiple hookworms in the jejunum and ileum. Hookworms caused the acute intestinal bleeding. The patient responded well to albendazole. Hematochezia was markedly ameliorated after eliminating the parasites. Hence, hookworm infection should be considered in the differential diagnosis of a patient with obscure gastrointestinal bleeding. Capsule endoscopy may offer a better means of diagnosis for intestinal hookworm infections.
\end{abstract}

Key words: Ancylostoma duodenale, Necator americanus, hookworm, acute intestinal bleeding, capsule endoscopy, nephrotic syndrome, pulmonary embolism

\section{INTRODUCTION}

Hookworm infections, mainly caused by Necator americanus and Ancylostoma duodenale, are most commonly transmitted through contact with contaminated soil; especially, fecal-tooral transmission is much more common than penetration of the skin $[1,2]$. This disease mainly occurs in resource poor communities in the developing countries. Hookworm infection is not a common cause of acute gastrointestinal bleeding. Common symptoms include abdominal pain, flatulence, nausea, diarrhea, iron deficiency anemia, and hypoproteinemia [3], which are nonspecific. It's a treatable disease and results in complete recovery. Diagnosis is traditionally established by stool examinations. Sometimes unexplained eosinophilia may be a major clue usually in mild infection cases. Here, we report a rare case of acute intestinal bleeding caused by hookworm infection, during the treatment with steroids and anticoagulants, which was diagnosed by capsule endoscopy.

\footnotetext{
- Received 2 May 2017, revised 31 May 2017, accepted 19 June 2017.

*Corresponding author (liuh0618@163.com)

(C) 2017, Korean Society for Parasitology and Tropical Medicine

This is an Open Access article distributed under the terms of the Creative Commons Attribution Non-Commercial License (http://creativecommons.org/licenses/by-nc/4.0) which permits unrestricted non-commercial use, distribution, and reproduction in any medium, provided the original work is properly cited.
}

\section{CASE RECORD}

A 46-year-old male complaining of edema for 2 months and exertional dyspnea and chest pain for 2 weeks was admitted to our hospital. The patient denied abdominal pain, nausea, and loss of appetite. He had no history of diabetes mellitus, hypertension, or malignancy. Initial laboratory studies showed that his hemoglobin level was $11.1 \mathrm{~g} / \mathrm{dl}$. Urinalysis revealed yellow urine with proteinuria 7,588 mg per day. CT angiogram (CTA) of lungs showed multiple pulmonary embolism (Fig. 1). The patient was given warfarin for pulmonary embolism, glucocorticosteroids and tarcrolimus for primary nephrotic syndrome. After 10 days, the patient presented with acute hematochezia and dizziness without obvious abdominal pain. The gastrointestinal bleeding did not stop when warfarin was stopped and treated with Vitamin K. On physical examinations, the patient's vital signs were stable on admission. However, his blood pressure was falling quickly after he got admitted with acute gastrointestinal bleeding. His abdomen was soft. Bowel sounds were normoactive. Several external hemorrhoids were evident but without bleeding. Laboratory results were as follows: hemoglobin $11.1 \mathrm{~g} / \mathrm{dl}$ on admission, fell to $3.8 \mathrm{~g} / \mathrm{dl}$ in his 20 days of gastrointestinal bleeding; white blood cell count, $7.05 \times 10^{9} / \mathrm{L}$; platelets, $408 \times 10^{9} / \mathrm{L}$; eosino- 


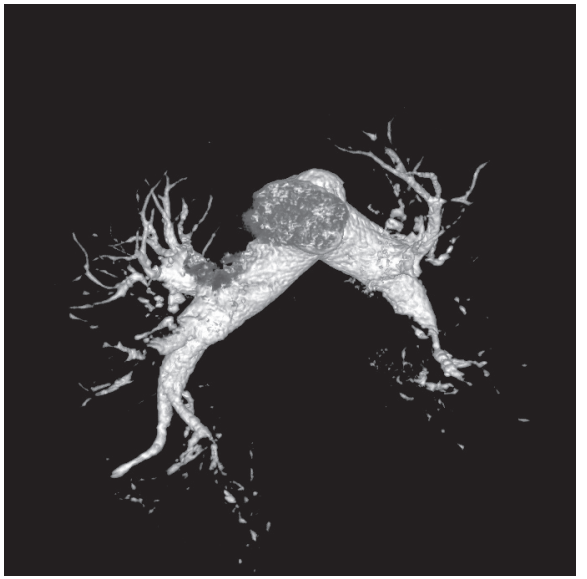

Fig. 1. CT angiogram showing multiple pulmonary embolism.

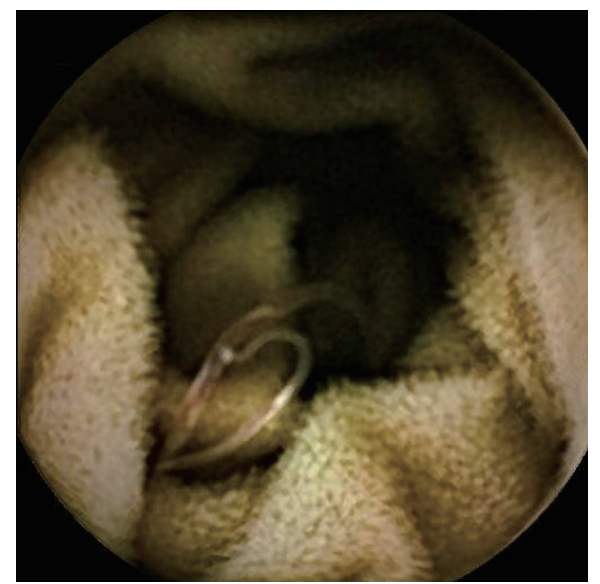

Fig. 3. Capsule endoscopy identified hookworms in the patient's intestine.

phils, $0.44 \times 10^{9} / \mathrm{L} ; 7,588 \mathrm{mg}$ proteinuria per day; albumin $14.9 \mathrm{~g} / \mathrm{L}$; international normalized ratio (INR) 1.05, partial thromboplastin time $13.5 \mathrm{sec}$, ferritin $707.8 \mathrm{ng} / \mathrm{ml}$; IgE 1,554 $\mathrm{ng} / \mathrm{ml}$; and transferritin $0.79 \mathrm{~g} / \mathrm{L}$. Other results, including colonoscopy, were unremarkable.

The patient underwent a CTA of the abdomen, which showed multiple deep vein thrombosis, but without definite evidence of gastrointestinal bleeding, retroperitoneal hematoma, or acute mesenteric ischemia (AMI). Gastroscopy showed hookworms in the duodenum, and the intestine is thought to be the source of bleeding (Fig. 2). A capsule endoscopy demonstrated fresh blood in the jejunum and multiple worm-like objects in the jejunum and ileum, all with a similar curvature and large "heads" (Figs. 3, 4). The images were concerning for a hookworm infection.

The patient was given 40-80 mg of methylprednisolone dai-

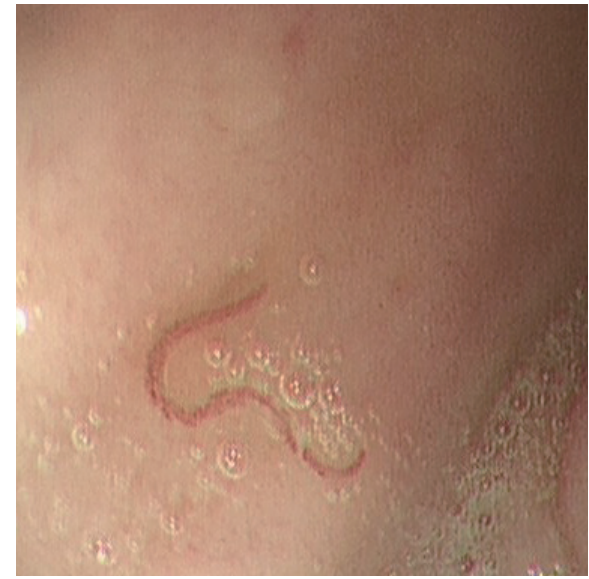

Fig. 2. Gastroscopy showing red worms swimming in the duodenum.

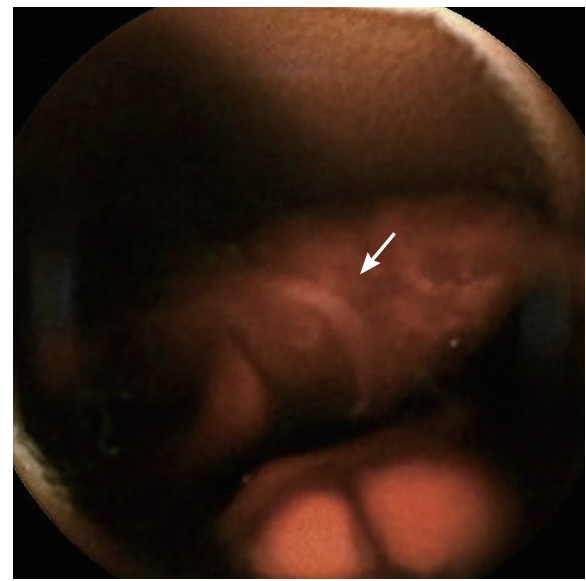

Fig. 4. Another capsule endoscopy identified a hookworm (arrow) in the patient's intestine.

ly and $1 \mathrm{mg}$ of tarcrolimus twice daily in hospital for the primary nephrotic syndrome. His warfarin's dose was dependent on INR; the target INR was 1.8-2.8. Warfarin was stopped when gastrointestinal bleeding occurred, and was given again after his bleeding stopped after half a month. For the hookworm infection, the patient was treated with multiple blood transfusion, iron, folic acid, and $400 \mathrm{mg}$ of albendazole twice daily for 3 days. Then, his gastrointestinal bleeding subsided. His hemoglobin at discharge was $6.6 \mathrm{~g} / \mathrm{dl}$. His proteinuria was negative after treatment with methylprednisolone and tarcrolimus for 10 days. The patient reduced methylprednisolone and tarcrolimus regularly, and insisted on taking warfarin. Six months later, his hemoglobin level was $12.8 \mathrm{~g} / \mathrm{dl}$, stool routine normal, proteinuria negative, and albumin $34.1 \mathrm{~g} / \mathrm{L}$. CTA of the lungs showed multiple pulmonary embolism recovered (Fig. 5). 


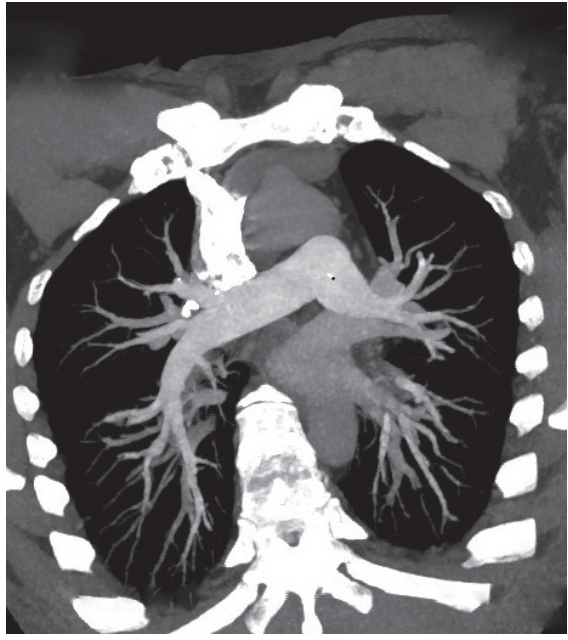

Fig. 5. CT angiogram after 6 months showing multiple pulmonary embolism recovered.

\section{DISCUSSION}

The hookworm was first described by an Italian physician, Dubini, in 1838 [4]. The disease is mainly caused by Necator americanus and Ancylostoma duodenale. Hookworms are transmitted through contact with contaminated soil, especially fecal contamination $[1,2]$. Most infected individuals present with chronic occult bleeding or asymptomatic. Acute gastrointestinal bleeding due to hookworm infections was rarely described previously. However, in developing countries especially in the tropics, worm infection should be considered an important cause of obscure acute gastrointestinal bleeding [5-7].

Evaluation of the jejunum using an endoscopy will result in more frequent diagnosis of hookworms as a cause of acute gastrointestinal bleeding which might have been classified as obscure gastrointestinal bleeding. Recently, several case reports detailed diagnosis of intestinal hookworm infections by capsule endoscopy [8-13]. In 2 of these, the patients' stool was positive for eggs. Four of the cases did not mention stool testing. In 1 case, endoscopy demonstrated the presence of hookworms, but the patient's stool samples were negative for eggs or larvae. Thus, they supported the opinion that examination of the stool is insensitive. Capsule endoscopy may offer a better means of diagnosis for intestinal hookworm infections. All of these patients presented with chronic bleeding or iron deficiency anemia [8-13], while our patient presented with very rare acute gastrointestinal bleeding and diagnosed by capsule.

Hookworm infection is a treatable disease and results in complete recovery. The 2 most commonly used drugs for treat- ment are mebendazole and albendazole, both of which are benzimidazole anthelmintic drugs. The drugs act by inhibiting microtubule polymerization in invertebrates, thereby killing adult worms. A single $400 \mathrm{mg}$ dose of albendazole is more effective than a single $500 \mathrm{mg}$ dose of mebendazole $[14,15]$. Three consecutive daily doses of either drug improve both cure and egg reduction rates $[16,17]$.

The nephrotic syndrome is a clinical complex characterized by a number of renal and extrarenal features. Essentials of diagnosis include proteinuria of $>3.5 \mathrm{~g}$ per $1.73 \mathrm{~m}^{2}$ per $24 \mathrm{hr}$ and hypoalbuminemia (serum albumin $<30 \mathrm{~g} / \mathrm{L}$ ). Renal biopsy is required to make the diagnosis and determine therapy in idiopathic nephrotic syndrome. However, this patient had no conditions for renal biopsy. One of the well-known complications of nephrotic syndrome is venous thrombosis. Hypercoagulability in these patients appears to be derived from urinary loss of anticoagulant proteins, such as antithrombin III and plasminogen, along with the simultaneous increase in clotting factors, especially factors I, VIII, and X. So, when acute gastrointestinal bleeding occurs, the possible condition of acute mesenteric ischemia (AMI) should be considered. This patient was treated with steroid and tacrolimus. The adverse effects of glucocorticoids include peptic ulcer with possible perforation, hemorrhage, and ulcerative esophagitis. Taking warfarin has the risk of bleeding. However, if not for warfarin, endoscopy could not show any ulcer, and CTA could not show any evidence of AMI, and we should consider some rare conditions such as infection of parasites. Capsule endoscopy offers a better means of diagnosis for intestinal hookworm infections.

\section{ACKNOWLEDGMENT}

This work was supported by a research grant (no. 81470947) from the National Natural Science Foundation of China.

\section{CONFLICT OF INTEREST}

We have no conflict of interest related to this study.

\section{REFERENCES}

1. Hotez PJ, Brooker S, Bethony JM, Bottazzi ME, Loukas A, Xiao S. Hookworm infection. N Engl J Med 2004; 351: 799-807.

2. Owen RL. Parasitic Disease. In: Sleisenger MH. Fordtran JS eds, Gastrointestinal Disease. Philadelphia, USA. WB Saunders. 
1993, pp 1211-1212.

3. Thomas V, Harish K, Tony J, Sunilkumar R, Ramachandran TM, Anitha PM. Colitis due to Ancylostoma duodenale. Indian J Gastroenterol 2006; 25: 210-211.

4. Dock G, Bass CC. Hookworm Disease. St. Louis, Missouri, USA. CV Mosby Company. 1910, pp 20-63.

5. Sharma BC, Bhasin DK, Bhatti HS, Das G, Singh K. Gastrointestinal bleeding due to worm infestation, with negative upper gastrointestinal endoscopy findings: impact of enteroscopy. Endoscopy 2000; 32: 314-316.

6. de la Riva H, Escamilla DG, Frati AC. Acute massive intestinal bleeding caused by hookworm. JAMA 1981; 246: 68 .

7. Wu FQ, Xu Y, Xia M, Ying GH, Shou Z. Hookworm anemia in a peritoneal dialysis patient in China. Korean J Parasitol 2016; 54: 315-317.

8. Seidelman J, Zuo R, Udayakumar K, Gellad ZF. Caught on capsule: iron-deficiency anemia due to hookworm infection. Am J Med 2016; 129: 167-169.

9. Nakagawa Y, Nagai T, Okawara H, Nakashima H, Tasaki T, Soma W, Hisamatu A, Anan J, Murakami K, Fujioka T. Comparison of magnified endoscopic images of Ancylostoma duodenale (hookworm) and Anisakis simplex. Endoscopy 2009; 41 (suppl): e189.

10. Chen YY, Soon MS. Endoscopic diagnosis of hookworm infection that caused intestinal bleeding. Gastrointest Endosc 2005; 62: 142 .

11. Rana SS, Bhasin DK, Sinha SK. Endoscopic diagnosis of chronic severe upper GI bleeding due to helminthic infection. Gastrointest Endosc 2008; 68: 1023.

12. Kalli T, Karamanolis G, Triantafyllou K. Hookworm infection detected by capsule endoscopy in a young man with iron deficiency. Clin Gastroenterol Hepatol 2011; 9: e33.

13. Wu IC, Lu CY, Wu DC. Acute hookworm infection revealed by capsule endoscopy. Endoscopy 2007; 39 (suppl): e306.

14. Keiser J, Utzinger J. Efficacy of current drugs against soil-transmitted helminth infections: systematic review and meta-analysis. JAMA 2008; 299: 1937-1948.

15. Vercruysse J, Behnke JM, Albonico M, Ame SM, Angebault C, Bethony JM, Engels D, Guillard B, Nguyen TV, Kang G, Kattula D, Kotze AC, McCarthy JS, Mekonnen Z, Montresor A, Periago MV, Sumo L, Tchuenté LA, Dang TC, Zeynudin A, Levecke B. Assessment of the anthelmintic efficacy of albendazole in school children in seven countries where soil-transmitted helminths are endemic. PLoS Negl Trop Dis 2011; 5: e948.

16. Steinmann P, Utzinger J, Du ZW, Jiang JY, Chen JX, Hattendorf J, Zhou H, Zhou XN. Efficacy of single-dose and triple-dose albendazole and mebendazole against soil-transmitted helminths and Taenia spp.: a randomized controlled trial. PLoS One 2011; 6: e25003.

17. Loukas A, Hotez PJ, Diemert D, Yazdanbakhsh M, McCarthy JS, Correa-Oliveira R, Croese J, Bethony JM. Hookworm infection. Nat Rev Dis Primers 2016; 2: 16088. 